Auch die von verschiedenen Forschern als eigentliche Primärerscheinung angenommene, regelmäßig vorkommende Verödung und Rückbildung der Stria vascularis ist wohl als eine sekundäre Äußerung der im ganzen Oktavussystem auftretenden degenerativen Prozesse, als eine neurotrophische Störung zu betrachten.

Was nun ferner die hinter jener Primärerscheinung liegende Ursache anbelangt, so kann vielleicht angesichts der Mitbeteiligung anderer sensorischer Apparate (s. oben) an eine fötale, von der Mundhöhle ausgehende Infektion gedacht werden (S. 134). Vielleicht führt auch das von K. auf mehreren Gehirnschnitten beobachtete Vorkommen rundlicher, nicht färbbarer, fast ganz durchsichtiger Gebilde auf eine Spur, mag es sich hierbei um Protozoen oder sonstige Parasiten mit dicker, die Tinktion verhindernder Membran oder um Endprodukte örtlicher Umbildungsprozesse handeln (S. 137).

Wie man sieht, konnte die eigentliche Ursache des charakteristischen Verhaltens der Tanzmäuse auch durch die schönen und in vieler Hinsicht grundlegenden Untersuchungen K.s nicht aufgedeckt werden, und es muß dahingestellt bleiben, ob eine erbliche, auf bestimmte Teile des Nervensystems selbst lokalisierte Entwicklungsschwäche vorliegt, die bei Eintritt der Funktion oder schon vorher ohne äußeren Anstoß zu einer frühzeitigen, nach van Lennep fortschreitenden Degeneration führt, oder ob die betreffenden Teile des Nervensystems normaler- oder abnormerweise eine geringere Resistenz gegen bestimmte Krankheitserreger besitzen oder vielleicht auch durch die erbliche Insuffizienz einer inneren Driise besonders stark beeinflußt werden. Jedenfalls weisen K.s Untersuchungen darauf hin, daß der funktionellen und wohl auch genetischen Zusammengehörigkeit der Bestandteile des Akustikussystems auch ein übereinstimmendes, über die Grenzen des Systems im ganzen wohl nur wenig hinausgreifendes Verhalten gegenüber deletären Einflüssen, eine Art von Organschwäche oder besser von regionaler Minderwertigkeit des Nervensystems entspricht. Die entwicklungsgeschichtlich und physiologisch komplexe Natur des Substastes, auf welches die letzte Ursache einwirkt, macht es auch dann, wenn die eigentliche Ursache selbst "einfach" ist, verständlich, daß nicht nur individuelle Verschiedenheiten im äußeren Verhalten der Tiere und in der Degeneration des Nervensystems, sondern auch UnregelmäBigkeiten in der Vererbung auftreten.

\title{
V. Haecker.
}

Gaupp, Ernst. Angust Weismann, sein Leben und Werk. Jena (F. Fischer) 1917, VIII u. $297 \mathrm{~S}$.

Es mag vielen unerwartet gewesen sein, daß gerade Gaupp, der hervorragende Morphologe und Reformator der Schädellehre, dem Biologen Weismann in einer mit ebensoviel sachlicher Beherrschung des Stoffes wie Wärme geschriebenen Biographie ein Denkmal gesetzt hat. Auch mir war es eine kleine Überraschung, als Freund Gaupp mir Ende 1915 seinen Plan mitteilte, einen in der Königsberger physikalisch-ökonomischen Gesellschaft gehaltenen Vortrag zu einer Erinnerungsschrift, insbesondere berechnet für die ehemaligen Schüler W eis man $\mathrm{ns}$, auszubauen, und mich um eine Äußerung bat, da er von einem ähnlichen, meinerseits beabsichtigten Unternehmen gehört habe.

Nun liegt aber gerade darin, daß die beiden Arbeitsgebiete sich zwar berühren, aber nicht vollkommen zusammenfallen, für den Autor einer Biographie ein gewisser Vorteil, und namentlich dann, wenn es sich um das 
Lebenswerk eines besonders auch auf theoretischem Gebiete hervorragenden Forschers handelt, wird der Biograph im allgemeinen freier dastehen, wenn er nicht durch seine eigenen Arbeiten veranlaßt war, zu den Theorien und ihren Wandlungen immer aufs neue Stellung zu nehmen. Noch ein anderer günstiger Umstand kam für Gaupp in Betracht. Gerade bei den Größten der Wissenschaft ist das Lebenswerk und die Persönlichkeit am wenigsten voneinander zu trennen, und fiur den, der über das erstere schreiben will, ist die persönliche Erinnerung ein unschätzbarer Vorteil. Gaupp bat aber jahrelang der Schar von jüngeren Morphologen und Biologen angehört, die das Glück hatten, mit dem Weismann-Wiedersheim-Gruberschen Familienkreise in enger Verbindung zu stehen und speziell mit Weismann haben ihn auch die musikalischen Interessen verbunden. So waren bei Gaupp zwei Voraussetzungen in ähnlicher Weise vorhanden, wie vor kurzem bei Boveri, der auf dem vorletzten, in Graz abgehaltenen internationalen Zoologenkongreß dem Begrïnder der Neapler Station in glänzender.Form einen tiefempfundenen Nachruf gewidmet hat.

Gelegentlich der Münchener Zoologenversammlung 1894 hat einer der Führer der älteren vergleichend-morphologischen Richtung geäußert, daß von Weismanns Arbeiten wohl nur die entwicklungsgeschichtlichen Untersuchungen über die Dipteren dauernden Wert behalten werden. Gerade diese Arbeiten sehen wir aber in Gaupps Biographie nur kurz erwähnt, ebenso wie die chemischen und histologischen Schriften aus den Jahren 1857-62. Etwas eingehender werden nur diejenigen Spezialarbeiten besprochen, welche die ersten Bausteine zu dem späteren Theoriengebäude darstellen: die Studien zur Deszendenztheorie (Saison-Dimorphismus, Raupenzeichnung, Axolotl-Experimente), die klassischen Daphnoidenstudien und die Untersuchungen über die Keimzellenbildung bei den Hydromedusen, Der eigentliche Schwerpunkt des Gauppschen Buches liegt aber in der Darstellung der historischen Entwicklung von Weismanns biologischen Theorien, und zwar unterscheidet Gaupp nach der Stellungnahme Weismanns zu den drei großen Teilfragen der Zuchtwahllehre - Variabilität, Vererbung, Auslese - drei Perioden. Die erste ist wohl vom Jahre 1868, in welchem die akademische Antrittsrede: "Über die Berechtigung der Darwinschen Theorie" erschien, bis zum Anfang der $80 \mathrm{er}$ Jahre zu rechnen und nach G. dadurch gekennzeichnet, daß $\mathrm{W}$. hier noch im wesentlichen auf dem alten darwinistischen Standpunkt steht, also neben der Naturzüchtung auch die Vererbung erworbener Eigenschaften als artbildenden Faktor gelten läßt. Von Darwin weicht er aber insofern ab, als die Variationen nicht richtungsund regellos sein sollen, sondern durch die "physische Natur" der Organismen beschränkt und in bestimmter Richtung gelenkt werden. In der zweiten, mit dem Jahr 1893 („Über die Vererbung") beginnenden Periode, die man wohl als Hauptperiode bezeichnen darf, wird die Lehre von der Kontinuität des Keimplasmas begründet, die Vererbungslehre mit den Ergebnissen der Zellen-, Befruchtungs- und Reifungslehre verbunden und in der verschiedensten Weise für die Zuchtwahllehre und gegen Lamarck Stellung genommen. "Das starke Wort von der, Allmacht der Naturzüchtung' drückt den Überschwang persönlicher Hingabe aus, mit der Weismann in dieser Zeit für die Zuchtwahllehre und gegen ihre Angreifer eintrat." In der dritten Periode, die die letzten 20 Jahre von 1895 an umfaßt, tritt an Stelle des aufgegebenen Lamarckschen Umwandlungsprinzips das orthogenetische Prinzip der Germinalselektion, „durch das zwar dem Selektionsprinzip an sich ein größerer Geltungsbereich zugesprochen wird, die Darwin-Wallacesche 
Zuchtwahllehre aber eine sehr erhebliche Einschränkung erfährt". Nun gilt Personalselektion und Germinalselektion.

Gaupp, der entsprechend seiner eigenen stark historisch gerichteten Veranlagung in erster Linie die Entwicklung von Weis manns Anschauungen, ihre Begründung, ihre historische Bedingtheit und ihren mannigfachen Wechsel objektiv darzustellen bemüht war, hat alle drei Perioden mit der gleichen liebevollen Verehrung behandelt, ohne sich mit einer eingehenden Kritik zu befassen, und so ist es denn auch von anderer Seite (F. Lenz, Münch. Med. Woch. 13. XI. 17) als eine Art Mangel empfunden wurden, daß er auf die Hypothese der Germinalselektion in so ausführlicher Weise eingeht, ohne eigentlich hervorzuheben, daß sie schlechterdings nicht aufrecht erhalten werden könne. Ich möchte aber glauben, daß in dieser streng historischen Behandlung des Gegenstandes der besondere Reiz und der bleibende Wert des Gauppschen Buches liegt und daß es vor allem aus diesen Gründen Studierenden und angehenden Forschern nicht genug empfohlen werden kann. Denn bei dem geringen Raum, den in biologischen Lehrbüchern und Vorlesungen wegen der Fïlle des zu behandelnden Stoffes eben die Geschichte der biologischen Theorien einzunehmen pflegt, ist es zu begrüßen, wenn in einem so klar geschriebenen Werke diese in pädagogischer Hinsicht außerordentlich wichtige Betrachtungsweise einem breiteren Leserkreis näher gebracht wird.

Ganz ohne Kritik ist freilich, wie zu erwarten, auch Gaupps Darstellung nicht geblieben. Sie richtet sich an einzelnen Stellen nicht nur gegen Weismanns Spekulationen (z. B. S. 154), sondern auch gegen die unklare Anwendung gewisser Begriffe, so wenn z. B. statt des Ausdrucks "passiv wirkende Teile", womit von Weismann die durch ihre bloße Anwesenheit wirkenden Merkmale (Färbung, Chitinskelette, Dornen der Pflanzen) bezeichnet werden, wegen der Ähnlichkeit mit dem ganz bestimmten Rouxschen Begriffe: "passiv funktionierende" Gewebe (d. i. Stützsubstanzen) der Name "apraktisch" vorgeschlagen (S. 93), und danach für die „,nützlichen Teile" folgende Einteilung gegeben wird (S. 279):

I. Eupraktisch:

a) aktiv funktionierend,

b) passiv funktionierend.

II. Apraktisch.

Noch an einer anderenStelle (S. 278) findet Gaupp in seinem Gerechtig. keitsgefühl scharfe Worte der Kritik und zwar gegenüber Rádl, dem Verfasser der im übrigen gewiß verdienstvollen "Geschichte der biologischen Theorien ". G. tadelt nicht nur den gehässigen Ton, in welchem Rádl von Weismann spricht, sondern hebt besonders noch hervor, daß Rádl u. a. die vielen experimentellen Arbeiten Weis manns unbekannt geblieben sind.

V. Haecker. 